\section{Effect of Traditional Cardiovascular Risk Factors on the Independent Relationship of Leptin with Atherosclerosis in Rheumatoid Arthritis}

To the Editor:

Leptin is an adipokine that regulates appetite and energy expenditure ${ }^{1}$. Both high and low leptin production can further increase cardiovascular (CV) risk $^{1}$. Leptin is also produced in inflamed joints and implicated in the pathophysiology of rheumatoid arthritis (RA) ${ }^{2}$.

Whether leptin increases $\mathrm{CV}$ risk in RA is currently uncertain. Two studies reported a lack of association between leptin concentrations and carotid artery intima-media thickness (cIMT) in RA ${ }^{3,4}$. Leptin concentrations were also found to be unrelated to coronary artery classification scores in $\mathrm{RA}^{5}$. However, we recently reported an independent relationship between leptin concentrations and surrogate markers of early atherogenesis in young patients with $\mathrm{RA}^{2}$. Importantly, in the present context, carotid artery plaque is a more reliable indicator of atherosclerosis than $\mathrm{cIMT}^{6}$.

In our present study, we examined the independent relationships of leptin concentrations with cIMT and plaque in 217 (112 black and 105 white) patients with RA. Because the production and effects of adipokines on $\mathrm{CV}$ risk depend on pathophysiological context ${ }^{1,2,7}$, we also determined whether the presence of conventional and nonconventional CV risk factors modified leptin concentrations and their associations with atherosclerosis.

All patients were receiving disease-modifying agents for rheumatic disease that included tumor necrosis factor- $\alpha$ blockade and rituximab in $3.7 \%$ and $1.2 \%$ of them, respectively. The Human Research Ethics Committee (Medical) from The University of the Witwatersrand in Johannesburg, South Africa, approved the protocol (approval number: M06-07-33) and each participant gave informed written consent.

Carotid ultrasound measurement methods and its reproducibility in our setting were previously reported ${ }^{7}$. Carotid artery plaque was defined as a focal structure that encroaches into the arterial lumen of at least $0.5 \mathrm{~mm}$ or $50 \%$ of the surrounding intima-media thickness value, or demonstrates a thickness of $>1.5 \mathrm{~mm}$ as measured from the media-adventitia interface to the intima-lumen interface ${ }^{8}$.

Leptin concentrations were measured using solid-phase sandwich ELISA (QuantikineHS, R\&D Systems Inc.). The lower detection limit was $7.8 \mathrm{pg} / \mathrm{ml}$, and the interassay and intraassay coefficients of variation were $4.4 \%$ and $3.2 \%$, respectively.

These characteristics were previously reported overall and in the different age quartiles in the present cohort ${ }^{2}$ : demographic features, lifestyle factors, anthropometric measures, conventional metabolic risk factors, C-reactive protein concentrations, glomerular filtration rate (GFR), $\mathrm{CV}$ agents, and leptin concentrations. The median (interquartile range: IQR) erythrocyte sedimentation rate was $12(5-28) \mathrm{mm} / \mathrm{h}$.

The mean (SD) cIMT and plaque prevalence were $0.709(0.109) \mathrm{mm}$ and $40.6 \%$, respectively. Leptin concentrations in all patients and relevant subgroups $^{7}$ are given in Table 1 . In univariate analysis, leptin concentrations were larger in black compared to white participants, and in patients with compared to those without major conventional $\mathrm{CV}$ risk factors and overall and abdominal obesity. Each of these differences was no longer significant in confounder and/or mediator-adjusted analysis ${ }^{2}$.

Leptin concentrations were unrelated to cIMT in all patients and in those with and without conventional or nonconventional risk factors (data not shown). Table 1 also shows the relationships of leptin concentrations with carotid artery plaque. In univariate analysis, leptin concentrations were consistently unassociated with plaque. The number of major conventional CV risk factors affected the leptin concentrations-plaque prevalence association (interaction $\mathrm{p}=0.02$ ). In adjusted analysis, a 1-SD increment in leptin concentrations increased the OR for plaque 2.75-fold in patients with major conventional CV risk factors; the respective OR $(95 \% \mathrm{CI})$ was $2.64(1.18-5.92, \mathrm{p}=0.01)$ in patients with $2(\mathrm{n}=40)$ or $\geq 3(\mathrm{n}=10)$ major risk factors. Patients with major $\mathrm{CV}$ risk factors had a larger body mass index (BMI) and lower GFR than those without these risk factors [median $(\mathrm{IQR})=27.7(24.0-32.8)$ vs $24.0(21.7-28.5) \mathrm{kg} / \mathrm{m}^{2}, \mathrm{p}=0.0001$, and 94
(81-111) and $102(89-123) \mathrm{ml} / \mathrm{min}, \mathrm{p}=0.05$, respectively]. As shown in Table 2, the leptin concentrations-plaque prevalence relation was driven by the GFR and BMI. This suggests an effect of leptin on carotid plaque that is mediated by increasing adiposity and renal impairment. Alternatively, our results may conceptually represent a compensatory increase in leptin production that is targeted at reducing atherosclerosis 5

Whereas in our previous study, leptin concentrations were associated with endothelial activation among patients aged $<50$ years ${ }^{2}$, a relationship of leptin with plaque was not found in the respective group in our present investigation. This indicates that whereas leptin may be involved in early atherogenesis, this adipokine may not contribute to advanced atherosclerosis in young patients with RA. Interestingly, the leptin-endothelial activation relationship was also obesity-driven in $\mathrm{RA}^{2}$.

Several recorded characteristics were non-normally distributed in our present investigation. When we repeated the analyses using log-transformed variables in the mixed regression models, the results were materially unaltered (data not shown).

We found an effect of leptin on carotid artery plaque that is dependent on the number of major conventional risk factors, which were identified in $70 \%$ of participants. Indeed, Rho, et al also reported that the effect of leptin on coronary atherosclerosis may be mediated through interactions with other $\mathrm{CV}$ risk factors in $\mathrm{RA}^{5}$. Plaque represents advanced atherosclerosis 6 that is strongly associated to coronary heart disease risk factors and incident coronary heart disease in both non-RA and RA subjects ${ }^{9,10}$. Leptin may contribute to the reported link between conventional risk factors and enhanced $\mathrm{CV}$ risk in RA. Consideration of leptin concentrations may improve $\mathrm{CV}$ risk stratification in patients with RA. These findings merit further investigation in future longitudinal studies.

Supported by the South African Medical Research Council (grant MRC2008_DES) and National Research Foundation.

PATRICK H. DESSEIN, MC, FCP(SA), FRCP(UK), PhD; LINDA TSANG; ANGELA J. WOODIWISS, PhD, Cardiovascular Pathophysiology and Genomics Research Unit, School of Physiology, Faculty of Health Sciences, University of the Witwatersrand; AHMED SOLOMON, MBBCh, FCP(SA), Department of Rheumatology, Charlotte Maxeke Johannesburg Academic Hospital, Faculty of Health Sciences, University of the Witwatersrand, Johannesburg, South Africa. Address correspondence to Prof. P.H. Dessein, P.O. Box 1012, Melville 2109, Johannesburg, South Africa. E-mail: dessein@telkomsa.net

\section{REFERENCES}

1. Koh KK, Park SM, Quon MJ. Leptin and cardiovascular disease: response to therapeutic intervention. Circulation 2008;117:3238-49.

2. Dessein PH, Norton GR, Woodiwiss AJ, Tsang L, Solomon A. Age impacts on the independent relationships of leptin with cardiometabolic risk and surrogate markers of enhanced early atherogenesis in black and white patients with rheumatoid arthritis: a cross-sectional study. Rheumatol Int 2014;34:329-39.

3. Gonzalez-Gay MA, Gonzalez-Juanatey C, Rodriguez-Rodriguez L, Miranda-Filloy JA, Martin J, Llorca J. Lack of association between adipokines and ghrelin and carotid intima-media thickness in patients with severe rheumatoid arthritis. Clin Exp Rheumatol 2011;29:358-9.

4. Dessein PH, Norton GR, Badenhorst M, Woodiwiss AJ, Solomon A. Rheumatoid arthritis impacts on the independent relationships between circulating adiponectin concentrations and cardiovascular metabolic risk. Mediators Inflamm 2013;2013:461849.

5. Rho YH, Chung CP, Solus JF, Raggi P, Oeser A, Gedretsadik T, et al. Adipocytokines, insulin resistance, and coronary atherosclerosis in rheumatoid arthritis. Arthritis Rheum 2010;62:1259-64.

6. Solomon A, Norton GR, Woodiwiss AJ, Dessein PH. Obesity and carotid atherosclerosis in African black and Caucasian women with established rheumatoid arthritis: a cross-sectional study. Arthritis 
Table 1. Leptin concentrations (ng/ml) and the relationships with carotid artery plaque (per 1 SD increment) in all RA patients and subgroups. Univariate comparisons of leptin concentrations between subgroups were made using the Mann-Whitney U test and univariate associations of leptin concentrations with plaque in logistic regression models. Major risk factors comprised hypertension, dyslipidemia, smoking, and diabetes ${ }^{2}$.

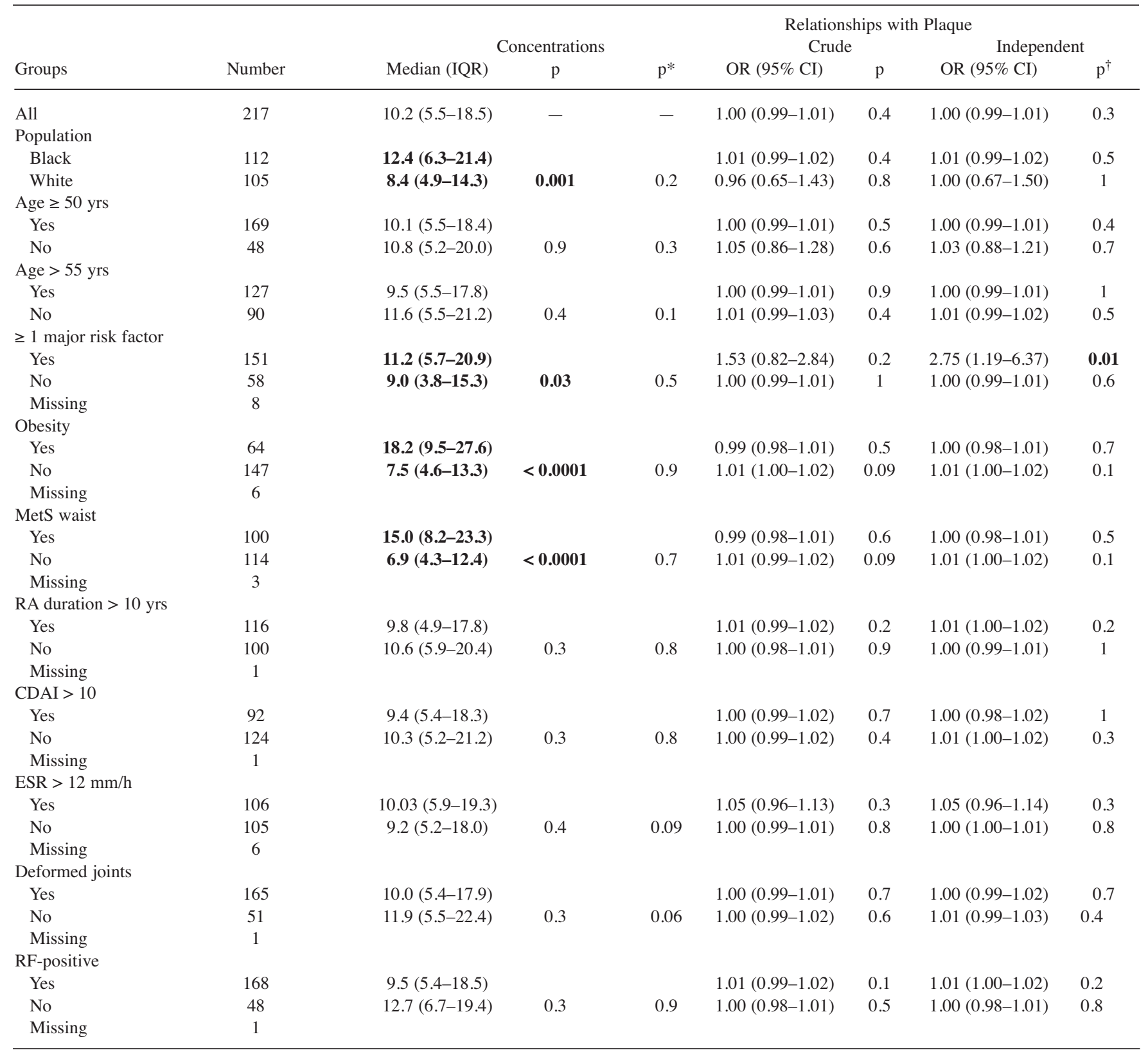

*p value for associations in age at disease onset, sex, race (except for in models by population grouping), body mass index, glomerular filtration rate, and cardiovascular drug use adjusted linear regression models. ${ }^{\dagger} \mathrm{p}$ value for associations in Framingham score, race, glomerular filtration rate, body mass index, and C-reactive protein adjusted logistic regression models. Significant associations are shown in bold face. RA: rheumatoid arthritis; IQR: interquartile range; MetS: metabolic syndrome; CDAI: Clinical Disease Activity Index; ESR: erythrocyte sedimentation rate; RF: rheumatoid factor.

Res Ther 2012;14:R67.

7. Dessein PH, Tsang L, Norton GR, Woodiwiss AJ, Solomon A. Retinol binding protein 4 concentrations relate to enhanced atherosclerosis in obese patients with rheumatoid arthritis. PLoS One 2014;9:e92739.

8. Touboul PJ, Hennerici MG, Meairs S, Adams H, Amarenco P, Bornstein N, et al. Mannheim carotid intima-media thickness consensus (2004-2006). An update on behalf of the Advisory Board of the 3rd and 4th Watching the Risk Symposium, 13th and 15th European Stroke Conferences, Mannheim, Germany, 2004, and Brussels, Belgium, 2006. Cerebrovasc Dis 2007;23:75-80.

9. Dessein PH, Norton GR, Woodiwiss AJ, Joffe BI, Wolfe F. Influence of nonclassical cardiovascular risk factors on the accuracy of predicting subclinical atherosclerosis in rheumatoid 
Table 2. Relations of leptin concentrations (1 SD increment) with carotid artery plaque among patients with major conventional cardiovascular risk factors. Associations were determined in logistic regression models.

\begin{tabular}{lcc}
\hline Adjusted Characteristics & OR $(95 \%$ CI $)$ & $\mathrm{p}$ \\
\hline None & $1.53(0.82-2.84)$ & 0.2 \\
Framingham score & $1.58(0.83-3.02)$ & 0.2 \\
Framingham score, race & $1.78(0.90-3.52)$ & 0.1 \\
Framingham score, race, CRP & $1.80(0.90-3.58)$ & 0.1 \\
Framingham score, race, CRP, GFR & $\mathbf{2 . 0 7}(\mathbf{1 . 0 0 - 4 . 2 9 )}$ & $\mathbf{0 . 0 4}$ \\
Framingham score, race, CRP, BMI & $\mathbf{2 . 3 5 ( 1 . 0 6 - 5 . 2 1 )}$ & $\mathbf{0 . 0 3}$ \\
Framingham score, race, CRP, GFR, BMI & $\mathbf{2 . 7 5 ( 1 . 1 9 - 6 . 3 7 )}$ & $\mathbf{0 . 0 1}$
\end{tabular}

Significant relations are shown in bold face. CRP: C-reactive protein; GFR: glomerular filtration rate, BMI: body mass index.

arthritis. J Rheumatol 2007;34:943-51.

10. Evans MR, Escalante A, Battafarano DF, Freeman GL, O'Leary

DH, del Rincon I. Carotid atherosclerosis predicts incident acute coronary syndrome in rheumatoid arthritis. Arthritis Rheum 2011;63:1211-20.

J Rheumatol 2014;41:10; doi:10.3899/jrheum.140315 